\title{
Estrategias para la participación de los usuarios en la producción de contenidos de tres cibermedios de referencia: BBC.co.uk, NYT.com y TheGuardian.com
}

\author{
Xosé López GARCÍA \\ Universidad Santiago de Compostela \\ xose.lopez.garcia@usc.es \\ Alba Silva RodríGuEZ \\ Universidad Santiago de Compostela \\ albasilvarodriguez@gmail.com
}

Recibido: 5 de diciembre de 2014

Aceptado: 8 de mayo de 2015

\begin{abstract}
Resumen
La gestión de la participación con criterios periodísticos se ha convertido en uno de los grandes desafíos de los principales cibermedios que presumen de ofrecer contenidos de calidad. El análisis de la $B B C$, The New York Times y The Guardian muestra vías para combinar la capacidad de algunos usuarios para aportar datos y perspectiva sobre algunos temas de actualidad y la garantía de la verificación profesional para asegurar piezas informativas veraces. $\mathrm{La} B B C$ ha establecido un modelo muy definido que da prioridad a la mediación profesional para verificar todas las aportaciones de los usuarios. Los análisis de caso muestran como cada medio diseña la gestión de la participación mediante modelos claramente diferenciados. El camino de la experimentación domina el panorama de los medios analizados.
\end{abstract}

Palabras clave: Cibermedios, participación de los usuarios, calidad de los contenidos, ciberperiodismo.

\section{Strategies for users' participation in the production of contents of three reference cybermedia: BBC.co.uk, NYT.com and TheGuardian.com}

\begin{abstract}
Management of public participation with journalistic standards has become one of the greatest challenges of the leading online media that claim to provide quality content. An analysis of the $B B C$, The New York Times and The Guardian shows ways to combine the ability of some users to provide data and perspective about some current issues, as well as ensuring professional verification in order to guarantee accurate and verified news stories. The $B B C$ has established a highly defined model that gives priority to professional mediation in order to verify all contributions from the users. Study cases show how each media designs management participation through very clearly differentiated models. Experimentation dominates the scene in the media analyzed.
\end{abstract}

Keywords: Cybermedia, user participation, quality of content, cyberjournalism.

\section{Referencia normalizada}

LÓPEZ GARCÍA, Xosé y SILVA RODRÍGUEZ, Alba (2015): "Estrategias para la participación de los usuarios en la producción de contenidos de tres cibermedios de referencia: BBC.co.uk, NYT.com y TheGuardian.com". Estudios sobre el Mensaje Periodístico. Especial noviembre "Periodismo e información de calidad", págs.: 145-164. Madrid, Servicio de Publicaciones de la Universidad Complutense.

Sumario: 1. Introducción. 2. Estado de la investigación. 3. Hipótesis y metodología. 4. Análisis; 4.1. El modelo de la $B B C ;$ 4.2. The Guardian y el UGC; 4.3. El modelo de The New York Times; 4.4. Algunos parecidos y diferencias; 4.5. Algunos rasgos de cibermedios franceses y norteamericanos. 5. A modo de conclusión. 6. Referencias bibliográficas. 


\section{Introducción}

La participación de los ciudadanos en los medios de comunicación ha sido una constante histórica. Es cierto que en los medios que denominamos tradicionales (prensa, radio y televisión) los canales para la intervención en los contenidos han tenido muchas limitaciones, algunas de las cuales formalmente han quedado eliminadas con el paradigma comunicativo construido alrededor de internet. Pero no es menos cierto que las nuevas ventanas abiertas por los cibermedios y la existencia de herramientas para intervenir no garantizan ni una participación activa ni una correcta canalización de esta para la construcción de un discurso periodístico de calidad.

El actual mapa de cibermedios está caracterizado por la convivencia de medios tradicionales que crearon productos para el ciberespacio y una larga lista de iniciativas de medios que hemos convenido en denominar nativos digitales (los nacidos y pensados para la red, sin antecedentes directos en otros soportes). El resultado es un mapa complejo que solo podemos entender analizando la evolución del sector de la comunicación en las últimas dos décadas. Lo cierto es que ambos tipos de cibermedios forman parte de un ecosistema de medios que siguen desempeñando un papel central en la producción y socialización de la reconstrucción de la realidad a través de los contenidos periodísticos que difunden con más o menos participación de las audiencias.

Ciertamente, en la actual situación del mapa ciberperiodístico han confluido procesos sociales, como la implosión del interés y el conocimiento común; tecnológicos, como la convergencia de las industrias de la telecomunicación, la informática y los medios; y periodísticos, marcados por vulnerabilidades recientes y antiguas del periodismo de referencia (Mazzone, 2012: 15). El nuevo escenario tecnológico y comunicativo, marcado por la digitalización, ha modificado todos los procesos esenciales atribuidos al trabajo periodístico, es decir, la búsqueda, elaboración y difusión de los contenidos informativos (Salaverría y García Avilés, 2008: 32).

De las diferentes mudanzas introducidas en la práctica periodística, ha sido la participación de los usuarios una de las principales conquistas formales, en especial a la hora de establecer vías para un discurso más plural y con más voces. Las posibilidades de participación de los ciudadanos y las nuevas opciones de producción de contenidos que pusieron en sus manos las tecnologías actuales alimentaron el campo de experimentación en el campo de la comunicación de muchos para muchos. La ciudadanía mediatizada, que ha surgido con el despliegue de la imprenta y la alfabetización, se ha visto reforzada y ampliada de la mano de los componentes de difusión de la radio y la televisión, mientras ahora son los medios digitales los que contribuyen con sus rasgos concretos a esta evolución, influyendo en el modo social y cultural de participar (Dahlgren, 2012: 49). El éxito de las redes sociales generalistas ha acentuado la transformación del panorama de los medios y el papel de los ciudadanos en el nuevo escenario comunicativo.

Las expectativas creadas por los posibles cambios en las prácticas periodísticas, en esa alianza con las tecnologías actuales y la sociedad en red para alimentar la participación, han sido muchas. No obstante, los resultados no siempre han sido los deseados y no todos los modelos establecidos arrojan los mismos resultados. La apertura de 
la participación a los usuarios les ha permitido a muchos cibermedios impulsar significativamente su consumo en línea en base a una combinación de factores favorables, entre los cuales destacan los blogs (Mazzone, 2012: 57).

La adopción de herramientas de participación en la esfera mediática ha creado un nuevo concepto, el de "periodismo participativo", en el que los ciudadanos aportan contenidos a partir de las informaciones previamente elaboradas por los medios. Pero podemos distinguir diversos modelos de participación a partir del análisis de la interactividad. Por una parte, la interactividad selectiva, que se refiere a todo lo que tiene que ver con los modelos de personalización de las interfaces y de la adecuación de los contenidos a nivel formal de acuerdo con las preferencias de los usuarios; la interactividad participativa, centrada en la presencia y la utilización de dos de las herramientas básicas de participación, como son los comentarios y las redes sociales y, por último, la interactividad productiva, que alude a las colaboraciones de los lectores que aportan una elaboración de auténtica información propia (Suau, 2011). Es precisamente en esta última categoría donde centramos nuestro interés en este trabajo.

Junto al concepto de "periodismo participativo" se ha consolidado el de "contenido generado por los usuarios" para describir el material creado por el público, en el contexto noticioso y en el que no lo es (Wardle y Williams, 2010). El debate terminológico en torno a la manera más adecuada de hablar de las aportaciones de los usuarios a los contenidos de los medios ha dado lugar a posturas encontradas entre investigadores. Muchos estudiosos se han concentrado en hablar de un único tipo de participación de la audiencia, el periodismo ciudadano, obviando la sinergia que existe entre este y el periodismo participativo. En los últimos años tal correlación ha derivado en el interés por el concepto de "CGU (Contenido Generado por los Usuarios) en las noticias (Avilés y Carvajal, 2008; Domingo et al., 2007; Ornebring, 2008; Paulussen y Ugille, 2008 o Thurman, 2008) con estudios que han examinado los tipos de contenido generado por los usuarios en diferentes salas de redacción y que han puesto de relieve la necesidad de examinar el grado en el que los usuarios tienen control sobre el contenido que generan y los tipos de contenidos que se les da la oportunidad de crear. Thurman (2008), a partir de un estudio de los periódicos online británicos en 2008, pudo diferenciar hasta seis tipos de participación de los usuarios: 1) encuestas en donde se anima a los usuarios a escoger una votación binaria o múltiple; 2) "have your say" (lo que dicen los usuarios) en el que se ofrece un canal en el que se publican opiniones de la audiencia en relación a algún tema seleccionado por la redacción; 3) chats, en los que se crea la conversación en tiempo real; 4) "Q\&As", relacionado con entrevistas con usuarios en las que se incorporaban preguntas sugeridas por los usuarios; 5) blogs; 6) tablones de mensajes. Algunos elementos de la estructura planteada por Thurman siguen vigentes en la actualidad en las principales empresas mediáticas y, concretamente, en las que exponemos en este análisis. Lo podemos ver en los siguientes ejemplos que muestran los espacios dedicados al debate y a la reflexión bajo la etiqueta "have your say" a la que se acogen los medios británicos. 
News $\mid$ Sport $\mid$ Comment $\mid$ Culture $\mid$ Business $\mid$ Money $\mid$ Life \& style
Life \& style Starters

Have your say

Tell us what you think

The Guardian, Friday 5 August 201123.01 BST

I was excited when I read the words "I own the Back To The Future car"

on last week's cover, only to be disappointed by an Experience article

about someone who simply owns a DeLorean. Please take more care

with the definite article.

Jessica Treen

Manchester

In What I'm Really Thinking, the high-flying wife's travelling for work is

suggested as the explanation for the husband's infidelity. Would his own

extensive travelling ("weekends in Italy or stopovers in Shanghai")

similarly be used to excuse his wife cheating. I wonder?

Roona Simpson

Glasgow

I'm really thinking, "How nice that you no longer feel guilty." However, the real thoughtless one is the husband. He should have the balls to tell his wife, and if she's OK with it, then fine, go ahead with your "relationship".

Though after 15 years without an "I love you", I wouldn't feel so smug.

And no, my husband hasn't had an affair - sometimes I'm just fed up with the deceit of people

Jenny Hagan

Liverpool

Gráfico 1: Sección de "Have your say" de The Guardian.

Fuente: Captura de pantalla de la web de The Guardian

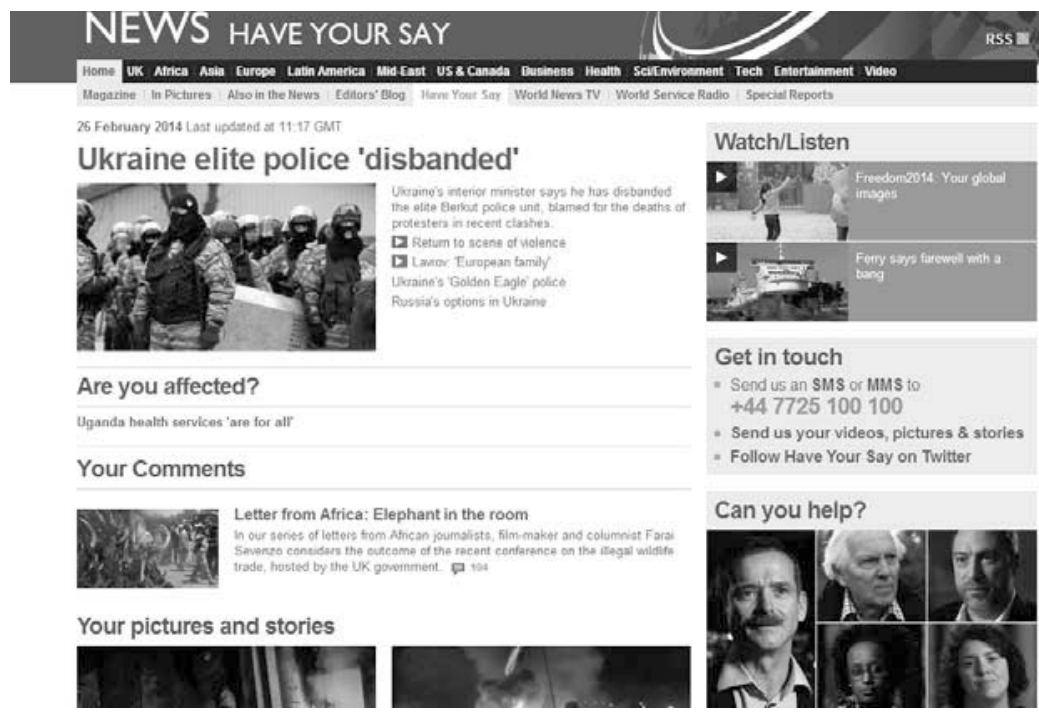

Gráfico 2: Sección de "Have your say" de la $B B C$.

Fuente: Captura de pantalla de la web de la $B B C$

La diversidad de materiales que el usuario puede enviar al medio nos lleva a concordar con la definición propuesta por McKenzie et al. (2012) al entender el UGC como aquel "contenido que es voluntariamente desarrollado por un individuo distri- 
buido a través de una plataforma online". Esta definición general nos permite encajar la diversidad de información que puede recibir el medio del usuario y que McKenzie clasifica como textual, audio, imágenes, videos y producciones multimedia producidas por individuos y distribuidas en la red como contenido creativo.

Pese a todo, el uso y consumo de medios digitales no parece que deba justificar taxativamente el empleo de modelos que no permitan la aplicación de los principios básicos del periodismo. De ahí la necesidad de analizar aquellos modelos que se consideran más acordes con la aplicación de la preceptiva periodística para conseguir un periodismo de calidad.

\section{Estado de la investigación}

La investigación sobre la participación en los cibermedios ha centrado varios proyectos de investigación y algunos aspectos han sido recogidos en documentos recientes (es el caso de la tesis doctoral de Mikel Iturbe (Pamplona, 2012) o de los libros de Natalia Raimondo (2012) y Elvira García de Torres (2012), entre otros. Los equipos en los que han participado estas dos autoras han dado continuidad científica a investigaciones sobre la interactividad, que en las últimas décadas del siglo XX contó con trabajos de Bretz (1983) y Jensen (1998), entre otros, y sobre la participación y la construcción de la actualidad en los cibermedios, que en la última década ha contado con trabajos de Deuze (2005), Rost (2006), Boczkowski (2006), Domingo (2007), Orihuela (2008), Masip et al. (2010), y Canavilhas (2011), entre otros.

El especial de Communications -The European Journal of Communication, en su volumen 37, número 3 de 2012-, fruto de un proyecto europeo Cost coordinado por Stak y Lunt, es una de las últimas aportaciones científicas al estado de la cuestión. Pero no es la única, sino solo una muestra más del interés que la interactividad, con las líneas de investigación y corrientes que ha abierto, ha despertado entre la comunidad científica de las ciencias sociales. La necesidad de buscar las mejores fórmulas para que la participación ordenada permita un periodismo de mayor calidad anima las últimas investigaciones, entre las que está el proyecto que lleva adelante el grupo Novos Medios en estos momentos ${ }^{1}$.

\section{Hipótesis y metodología}

La hipótesis central de la investigación que ha hecho posible este trabajo es que hay alternativas periodísticas para combinar la participación ordenada de los usuarios en los cibermedios y la aplicación de los principios periodísticos que aseguran una mediación profesional que cumple con los preceptos básicos de la profesión. El objetivo de la mayoría de esas alternativas consiste en convertir la participación en colaboración. De hecho, la práctica periodística nos muestra varios caminos, a los que debemos añadir sugerencias formuladas desde la investigación universitaria, que buscan

1 Esta comunicación incluye los datos recogidos en la fase de análisis del contexto periodístico y su metamorfosis realizado en el marco del proyecto de investigación del Ministerio de Economía y Competitividad "Innovación y desarrollo de los cibermedios en España. Arquitectura de la interactividad periodística en dispositivos múltiples: formatos de información, conversación y servicios. Referencia: CSO2012-38467-C03-03. 
mejoras en algunos de los principales modelos que aplican algunos de los medios digitales de referencia.

La metodología para la elaboración de esta investigación ha consistido en un seguimiento de los modelos de participación de los cibermedios BBC.com, NYT.com y TheGuardian.com, así como de varios medios norteamericanos y franceses que representan algunos de los modelos de cibermedios editados en esos países. El análisis de los elementos que caracterizan el modelo y el seguimiento durante dos semanas de los principales temas en los que ha habido participación relevante de los usuarios nos ha aportado datos para un estudio interpretativo sobre las fortalezas periodísticas de los dos modelos. El estudio empírico permitió conocer las vías aplicadas para incorporar las aportaciones de los ciudadanos y, al mismo tiempo, garantizar la verificación de la información por parte de los periodistas. Algunos aspectos que caracterizan este funcionamiento las recogemos a continuación y proceden de las conclusiones de los citados análisis.

\section{Análisis}

\subsection{El modelo de la $B B C$}

La llegada de Internet ha obligado a todos los medios a revisar sus estrategias y a reformular sus sistemas de trabajo. La $B B C$, que no ha sido una excepción a esta tendencia, ha procurado, sin embargo, mantenerse fiel a sus principios básicos desde su fundación, que recogen la relación directa con la audiencia como una vía para generar más y mejor información. Para ello, construyó un modelo que garantiza que los usuarios participan en la fase previa a la publicación, sin que suponga una suplantación del papel del periodista (Iturbe, 2012: 177-178). El modelo asegura que se escuchan las voces que tienen algo que decir, mediante la aplicación de la técnica periodística, y evita convertir los comentarios en el lugar de recogida de los frutos de las hordas de hooligans que campan por las zonas donde se puede ofrecer un punto de vista en los grandes medios (Salaverría, 2012).

El modelo de participación que promueve la $B B C$ muestra que existe un planteamiento periodístico previo que especifica qué se pretende obtener con cada aportación. Sus principios editoriales, claros, definidos y compartidos por los periodistas, facilitan, a la hora de fijar orientaciones y establecer cribas, una guía imprescindible para agilizar el trabajo profesional (Iturbe, 2012: 222). De hecho, la BBC News abre comentarios únicamente en aquellas informaciones donde la contribución del público pueda ser relevante en su calidad de potencial testigo y no por su eventual opinión sobre el asunto tratado. El modelo, por tanto, prima más los testimonios que los comentarios (Salaverría, 2012).

La gestión de contenido generado por los usuarios (user-generated content o UGC) está explícitamente incluida en las directrices editoriales de la $B B C$. Según señala, la $B B C$ aprovechará el rango completo de contenido generado por los usuarios siempre y cuando este sirva nuestros propósitos de servicio público y sea acorde con los estándares de calidad que nuestros usuarios esperan de nudos en la internet [...] Todo espacio online donde se publica contenido generado por la audiencia debe tener un moderador capaz de eliminar contenido ilegal e impropio y debería tener normalmente 
un host para proporcionar una presencia visible y activa. También debe haber una persona identificada dentro de la división relevante que tome responsabilidad editorial por el contenido, asegurándose de que el espacio mantiene estándares globales apropiados de moderación y hosting ${ }^{2}$.

De este modo, la cadena británica asume el contenido producido por su público como propio. Las aportaciones de la audiencia se rigen por los mismos estándares que cualquier contenido creado por los mismos periodistas de la $B B C$. Además, el servicio online de la $B B C$ cuenta con un esquema de actuación periodística que regula las aportaciones de los usuarios y fija reglas estrictas de juego, permitiendo que el producto final que llega al consumidor se encuentre altamente elaborado. "Tanto la validación y control de los comentarios como la decisión de cómo y cuándo se decide abrir una noticia a esos mismos comentarios cuentan con un razonamiento periodístico que justifica la decisión" (Iturbe, 2012: 118).

La $B B C$ ha sido uno de los principales medios que ha defendido con más claridad la importancia del contenido generado por los usuarios. Quizás sea el proyecto piloto lanzado en el año 2005 "User Generated Content (UGC) Hub" uno de los puntos de inflexión del medio británico en este nuevo escenario. Coincidiendo con la puesta en marcha de esta iniciativa se perpetraron los atentados de Londres del 2005, algo que demostró que la participación de los usuarios en acontecimientos de última hora como suponían un valor añadido a la información. En solamente seis horas, la $B B C$ había recibido mil imágenes, cuatro mil mensajes de texto y veinte mil emails. A partir de la repercusión de dicho acontecimiento y del éxito que supuso este proyecto, la $B B C$ se ha preocupado por el contenido generado por sus usuarios de tal manera que ha creado un espacio especial para esto y unas condiciones de uso específicamente dirigidas a la participación de la audiencia en los contenidos del medio.

Uno de los ejemplos más destacados de los últimos tiempos tiene que ver con la noticia de la muerte de Margaret Thatcher y la repercusión que este hecho suscitó en la audiencia a través de la constante participación a través de los medios sociales. El medio británico apostó por presentar el contenido de forma visual a través de un mapa, con el apoyo de una plataforma de monitorización basada en la ubicación de terceros llamada Geofeedia, en el que se señaló la ruta que seguiría la procesión fúnebre. El equipo de la $B B C$ estableció un flujo de trabajo a través del cual se podían supervisar las plataformas de los medios sociales en tiempo real para identificar contenidos únicos e interesantes y conseguir permisos para republicar ese contenido en un mapa para la presentación a un público más amplio.

Esta innovadora iniciativa, que nunca antes se había intentado realizar, consiguió resultados muy alentadores para el equipo encargado de administrar el contenido generado por los usuarios de la $B B C$. Este evento supuso un aumento significativo en el número de visitantes al sitio web del medio británico.

2 El texto figura en la página web de la $B B C$. Aquí se recoge la traducción de unos párrafos. 


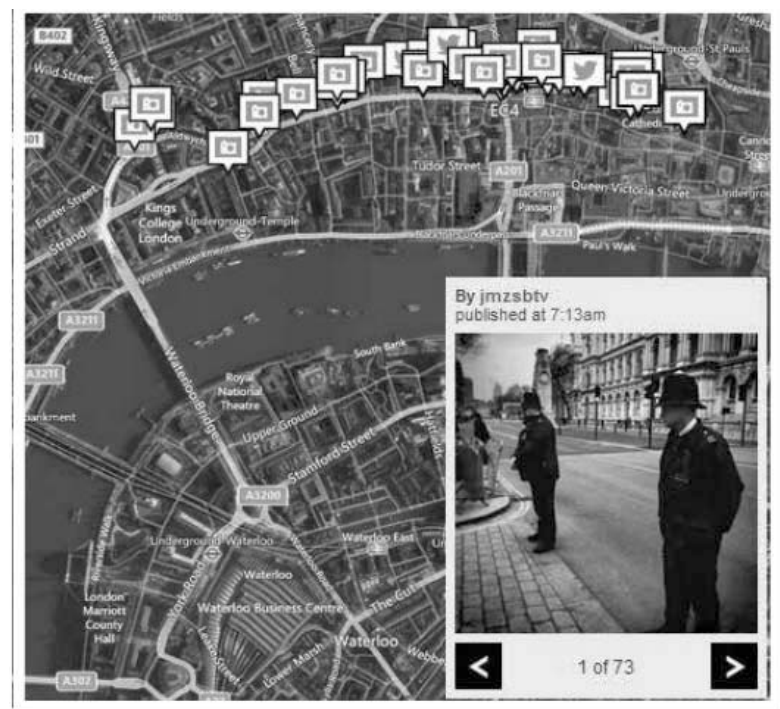

Gráfico 3: El funeral de Margaret Thatcher en tiempo real a través del contenido generado por los usuarios en directo. Fuente: Captura del sitio web de la $B B C$

La importancia de la gestión del contenido generado por los usuarios en la $B B C$ se manifiesta, entre otras cosas, en la aparición de herramientas que faciliten el trabajo de verificación de la información. En la $B B C$ se usa NewsWhip, Banjo, Reddit y muchas otras plataformas que trabajan con contenidos generados por los usuarios. Este medio opta, además, por verificar siempre el origen de las fuentes de la información proporcionada por los ciudadanos. En los casos en los que no se logra llegar a ellas, como ha ocurrido ya con algunos videos provenientes de Siria, casi imposibles de verificar, el equipo de CGU proporciona un lenguaje especial para los periodistas que trabajan con dicho material a partir de un ejemplo como el siguiente: "Precaución: Creemos que este material es original, pero debido a su naturaleza y origen, no podemos estar seguros. Cualquier uso debe incluir un texto de advertencia con leyendas como esta: "La $B B C$ no ha podido certificar la autenticidad total de este material, pero en base a comprobaciones adicionales, se cree que es genuino". Un total de 20 empleados (Turner, 2012) se encarga de examinar las contribuciones de la audiencia. Aunque inicialmente estaban centrados en imágenes y relatos de testigos enviados por correo electrónico a la $B B C$, en los últimos años el equipo de edición ha tenido que prestar atención a la participación de sus usuarios a través de redes sociales como Twitter, Youtube o Facebook. Los profesionales actúan en los medios sociales a partir de la herramienta de búsqueda, es decir, observando cuales son las tendencias en Twitter para localizar, posteriormente, las imágenes y tweets de contactos de confianza. La regla de oro, señala el equipo del medio británico encargado de esta labor, es conseguir el teléfono en el que se ha publicado ese material.

Algunos de los últimos reportajes de la $B B C$ que incluyen CGU (Contenido Generado por los Usuarios) fueron el tifón Haiyan, la muerte de Nelson Mandela o el uso de las armas químicas en Siria. 


\subsection{The Guardian y el UGC}

Uno de los medios más innovadores y uno de los pioneros en la inclusión del contenido generado por los usuarios en sus contenidos y en su web ha sido The Guardian.

El medio británico recoge en su web, en el punto 6 del apartado de términos y condiciones de uso, el conjunto de normas que rigen el contenido suministrado por la audiencia. En dichas pautas se recuerda al usuario la necesidad de aportar contenido original así como el derecho por parte del equipo de profesionales de The Guardian de "cortar, recortar, modificar o rechazar" tal publicación. Este breve apartado se complementa con un enlace a diez directrices basadas en normas legales y éticas que el medio británico pretende hacer llegar a los participantes de sus "áreas comunitarias". Observamos que, a pesar de hacer una clara apuesta por iniciativas y proyectos en los que el papel del usuario tiene un valor fundamental, todavía no existen unas normas de uso centradas en las aportaciones de los usuarios en los contenidos del medio, entendidas estas más allá de la simple interacción a través de comentarios. De hecho, la plataforma "Guardian Witness" en su página principal (witness.theguardian.com) en el apartado de "terms of service" no incorpora ninguna novedad al respecto y se limita a presentar las mismas condiciones de uso que en la web general. Sin embargo, las reglas de participación aparecen más especificadas en la sección de "FAQs", en donde se informa de las razones por las que un material enviado para "Guardian Witness" puede ser rechazado. Entre estas últimas se incluye el factor calidad en los contenidos multimedia o el hecho de que exista "alguna razón para dudar de la credibilidad de los contenidos". En las condiciones que publica The Guardian también se constata que el contenido de los usuarios irá acompañado del nombre del autor donde quiera que aparezca su contribución o que las aportaciones serán revisadas por el personal editorial del medio.

Los comienzos del medio británico en la explotación del contenido generado por sus usuarios tuvieron lugar a partir del año 2009. Durante ese período los esfuerzos del periódico en construir una base de datos de los gastos de los diputados británicos habían sido aclamados como un caso histórico para el periodismo participativo o de crowdsourcing. Para atraer a la audiencia frente a la labor que estaba ejerciendo en este terreno uno de sus principales competidores, The Daily Telegraph, The Guardian adoptó la fórmula de presentar la información a partir de una especie de entorno de juego para los usuarios. Se subieron a la web los 458.832 documentos en los que constaba la relación de gastos que miembros del Parlamento Británico pretendían cargar a las cuentas públicas para que los analizaran. Este proyecto desarrollado por Simon Willison se consideró un éxito tanto en lo que tuvo que ver con la consecución de más lectores y atención mediática como en la puesta en valor de la publicación de documentos como una herramienta para la innovación en la manera de contar las noticias. Sin embargo, esta experiencia también subrayó la imperante necesidad y la importancia de los procesos de verificación en el periodismo participativo.

La verificación, que forma parte de los elementos del periodismo, no solo resulta vital en el actual escenario, sino más importantes en un panorama donde la comunicación es ubicua y donde las estrategias de desinformación tienen un terreno abonado. La existencia de comunidades y redes sociales, muy importantes para el periodismo del tercer milenio, no pueden condicionar la verificación como esencia del periodismo. De ahí las iniciativas puestas en marcha en los últimos años para buscar renovadas herramien- 
tas que faciliten el trabajo de los periodistas. Una de las más interesantes ha sido la impulsada por diez instituciones europeas (incluyendo Yahoo e IBM) a partir del proyecto "EU Social Sensor" mediante la cual se desarrollan nuevas herramientas de software para ayudar a los periodistas a utilizar los medios sociales de manera más efectiva. Además de herramientas de verificación, surgen guías como "Verification Handbook" (Silverman, 2013) dirigidas a orientar a los periodistas ante este nuevo escenario.

Se trata de un campo de interés para el nuevo escenario periodístico, donde se precisan renovadas herramientas capaces de contribuir a la verificación y el descubrimiento de las falsedades que circulan como relatos de hechos verdaderos.

The Guardian cuenta con interesantes ejemplos de visualizaciones de datos, incluyendo mapas, gráficos y líneas de tiempo. Uno de los más emblemáticos por su repercusión y uno de los más recientes ha sido la representación de los registros de muertes por la guerra de Irak.

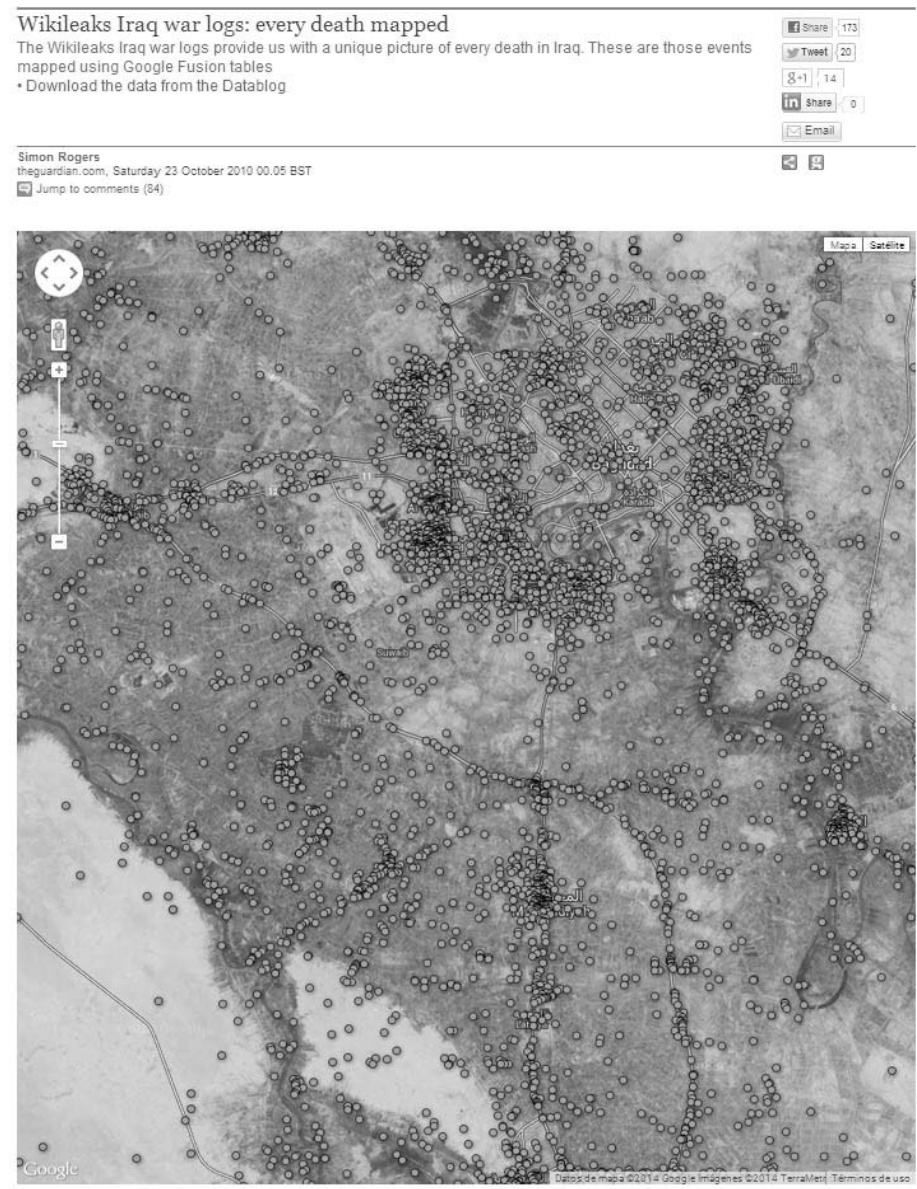

Gráfico 4: Visualización de datos de los registros de muertes de la guerra de Irak de The Guardian. Fuente: Captura de pantalla de la web de The Guardian 
Otros ejemplos más recientes y de similares características que ha lanzado el medio británico tienen que ver con los disturbios ocasionados en el Reino Unido en el verano de 2011 así como con el caso de Jimmy Mubenga en octubre de 2010.

En la actualidad, la categoría de "UGC (User Generated Content)" de la The Guardian se ubica en la sección Open Journalism y especialmente a partir de la plataforma Guardian Witness, un proyecto que constituye uno de los primeros ejemplos de periodismo participativo lanzado por una gran empresa mediática. Esta apuesta por el open journalism se acompaña de una aplicación móvil que permite a la audiencia estar cerca de las noticias locales y sociales que más le interesan. El principal objetivo de este proyecto consistía en la oportunidad de leer noticias que habitualmente quedaban fuera de la agenda mediática por falta de espacio o de interés. Es así como con The Guardian Witness nació una nueva manera de entender los contenidos. Una información generada por los usuarios, social, local y cultural. El nuevo usuario de The Guardian empezó a generar noticias, leerlas, difundirlas por las redes sociales desde su app móvil y web, etc.

\subsection{El modelo de The New York Times}

The New York Times, en el marco de sus principios editoriales, también estableció vías para permitir la participación de los usuarios. El NYT online incluye a la audiencia en sus directrices generales de funcionamiento. Sin embargo, el diario apenas habla sobre el papel del público en la creación de contenidos y su importancia en el periodismo del nuevo milenio. El NYT no habla en términos de comunidad y desarrollo colectivo. Por el contrario, el medio se centra en temas como las responsabilidades legales de sus lectores ${ }^{3}$.

El NYT implantó en 2011 un sistema de suscripción a sus contenidos digitales que limita el acceso a los usuarios y, consecuentemente, la participación ciudadana ${ }^{4}$. Sus políticas para dar voz a la audiencia y aprovechar sus aportaciones de cara a la oferta informativa están básicamente distribuidas entre la página de Términos de Servicio y las FAQ. La sección de Términos de Servicio incluye una subdivisión exclusivamente dedicada al contenido generado por los usuarios. En ella se indica que los usuarios no deben publicar en el espacio web del diario neoyorquino ningún tipo de contenido difamatorio, ofensivo o ilegal. Los usuarios tampoco pueden amenazar a ningún otro lector o interrumpir constantemente el diálogo mediante la difusión de spam. Asimismo, la discriminación racial, religiosa, política o sexual está prohibida.

Otra subsección habla del registro de usuarios y la seguridad del sitio web. Para crear una cuenta en NYTimes.com, hace falta suministrar un nombre de usuario y una

3 Las referencias al funcionamiento de la participación de los lectores en el diario The New York Times están basadas en el trabajo de fin de grado elaborado por Pablo Mayo Cerqueiro con el título "La gestión de la participación ciudadana en los medios digitales norteamericanos", en el grupo Novos Medios.

4 En 2011 el NYT introdujo un sistema de suscripción a sus contenidos virtuales que establece que los lectores solo pueden visitar la versión digital 20 veces al mes sin pagar. En: http://www.nytimes.com/2011/03/18/opinion/118times.html 
dirección de email. El medio advierte a los lectores de que deben responder por la actividad de sus cuentas: "Es responsable de todo uso o actividad en su cuenta en NYTimes.com, incluyendo la utilización de su cuenta por cualquier tercero autorizado por usted para emplear sus credenciales de acceso" (The New York Times, 2012).

Un último apartado en la página de Términos de Servicio habla de la comunicación entre los usuarios y The New York Times. Con todo, este no trata de la relación periodista-lector, sino que habla de privacidad y suscripciones a las actualizaciones del diario.

Según se señala en la página de preguntas frecuentemente formuladas de "Comments \& Readers' Reviews", los usuarios de The New York Times pueden publicar comentarios en diferentes piezas periodísticas o también pueden publicar críticas de filmes y libros. El diario expone que las aportaciones que busca son comentarios de interés para el tema de la pieza que cumplan con los estándares de calidad del NYT. Los comentarios son moderados a priori, esto es, en una fase previa a la publicación. Por lo tanto, si un comentario es filtrado por el equipo del NYT, generalmente la aportación no va a ser editada posteriormente. Asimismo, el medio afirma que "debido al volumen de comentarios de lectores, no podemos revisar decisiones individuales de moderación con lectores". Con todo, algunos usuarios poseen la etiqueta de "verifiedcommenters". Estos usuarios han demostrado que son respetuosos en sus comentarios y, por lo tanto, sus intervenciones son publicadas directamente.

Los usuarios también pueden denunciar contenido publicado por la audiencia para que sea revisado por el equipo de profesionales del diario estadounidense. Asimismo, el NYT anima sus lectores a emplear su nombre real para generar conversaciones más humanas y constructivas. Finalmente, el medio indica que si los usuarios detectan algún tipo de error en un artículo, o quieren proporcionar información sobre el evento en cuestión, estos deben ponerse en contacto con la redacción. El NYT proporciona diversas direcciones de correo electrónico, números de teléfono y faxes.

The New York Times ha destacado, al igual que los medios británicos que hemos señalado en los apartados anteriores, por desarrollar proyectos innovadores en el terreno de la participación de los usuarios. Paradigmático es el caso en el que el periódico neyorquino consiguió la agenda oficial del presidente de la Reserva Federal de Nueva York, Timothy F. Geither, la colgó en la web y le pidió a la gente que le ayudara a localizar reuniones o entrevistas con gente que pudiera explicar la actuación del mandatario durante la crisis bancaria.

Más recientemente, el periódico estadounidense cuenta la historia de Siria a través de los ciudadanos y sus vídeos. Una de las experiencias más ricas en este ámbito es la que se conoció como "Syria's War" a través de la que se presentaba el conflicto partiendo de vídeos de los ciudadanos de una manera que daba una visión de la vida cotidiana en tiempos de guerra y planteaba preguntas sobre lo que se veía. Cada vídeo incluía una sección llamada "lo que sabemos" y otra "lo que no sabemos" y un par de tweets relacionados.

\footnotetext{
5 http://www.nytimes.com/content/help/rights/terms/terms-of-service.html\#a

${ }^{6} \mathrm{http}: / /$ www.nytimes.com/content/help/site/usercontent/usercontent.html
} 


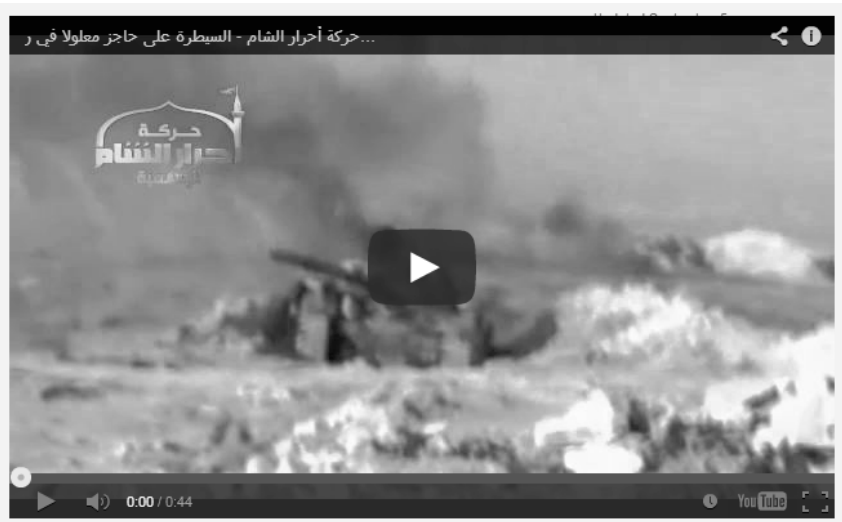

Video Suggests Cooperation Between Western- and Qaeda-Backed Rebels

Video posted to YouTube by accounts associated with both Islamist rebels and the Free Syrian Army showed the two groups working together to attack government positions in Malula, a town where Aramaic, the language spoken by Jesus Christ, is still used. The video was posted by the Islamist group Ahrar al-Sham, which the cameraman said coordinated the attack with the Qaeda-linked Nusra Front. The video suggests cooperation between the Western-backed Free Syrian Army and groups linked to Al Qaeda.

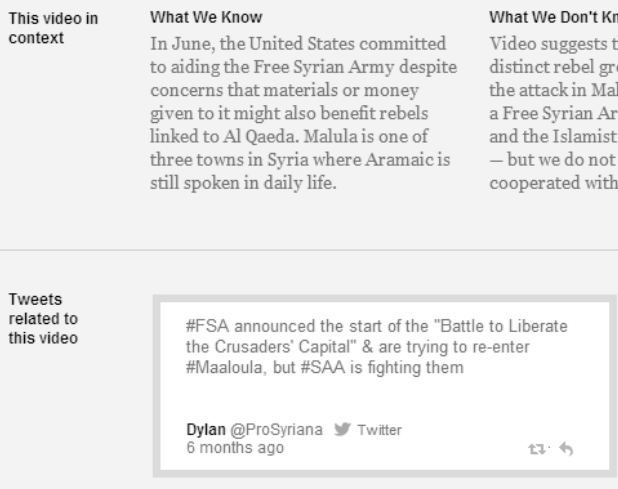

\section{If FACEBOOK Y TWITTER}

Other Videos

More footage of the battle was posted online by Ahrar al-Sham. Three more clips of the battle in Malula were posted online Wednesday by an account associated with the Free Syrian Army in Homs.

Gráfico 5: Sección de "Syria's War" del The New York Times, Fuente: Captura de pantalla de la web del The New York Times en su sección "Watching Syria's War

Jennifer Preston, editora de medios sociales de The New York Times, afirma en una entrevista publicada en The Myndset que a la hora de establecer una moderación o control de la información enviada por los usuarios se aplican las mismas normas que con cualquier otro contenido producido desde el medio. Preston afirma en una entrevista de Olivier Cimelière en The Myndset que se plantean varias preguntas antes de proceder a aceptar los contenidos: ¿Fue editado antes de su publicación?, ¿Quién lo creó?, ¿Cuál fue su motivo? En el caso de imágenes, por ejemplo, recalca que nunca se publicarían sin comprobar los derechos de autoría.

El procedimiento que sigue el The New York Times coincide, en gran medida, con las recomendaciones de la guía "Verification Handbook" (Silverman, 2013: 26). La fase de verificación combina técnicas humanas y tecnológicas. Las herramientas automáticas que surgen con el objetivo de agilizar el proceso se coordina con los siguientes pasos que deben realizar los periodistas del medio:

1. Procedencia: ¿Es original la pieza de contenido?

2. Fuente: ¿Quién sube el contenido a la Web? 
3. Fecha: ¿Cuándo ha sido creado el contenido?

4. Localización: ¿Dónde ha sido el contenido creado?

A pesar de las incógnitas que surgieron en un principio en torno a la amenaza en la calidad de la información generada por la audiencia e incluso a la identidad de los propios periodistas ante esta nueva situación, muchos autores no dudan en destacar los beneficios y ventajas que el open journalism conlleva. Johanna Vehkoo (2013) toma como referencia a Alan Rusbridger para destacar el valor que tiene la apertura de los procesos periodísticos para los lectores. Denomina a este fenómeno "mutualización" y la define, a partir de diez puntos, de la siguiente manera:

1. Fomenta la participación.

2. No se trata de una comunicación pasiva de "nosotros" a "ellos".

3. Anima a crear debate, publicar material o hacer sugerencias. Se puede seguir y también guiar, dirigir. Se puede involucrar a otros en los procesos previos de publicación.

4. Ayuda a formar comunidades de interés alrededor de temas, problemas o individuos.

5. Es accesible en la web y forma parte de este medio.

6. Se agrega y/o conserva el trabajo de otros.

7. Reconoce que los periodistas no son la única voz de la autoridad, experiencia e interés.

8. Aspira a mantener y reflejar diversidad así como a promover el intercambio de valores.

9. Reconoce que la publicación puede ser el principio de un proceso periodístico y no el fin.

10. Es transparente y abierto al cambio- incluyendo la corrección, clarificación y adición.

\subsection{Algunos parecidos y diferencias}

Los modelos de The Guardian y del NYT, aunque establecen garantías de control para que las aportaciones de los usuarios no empañen la calidad de las piezas, están poco desarrollado y poco implicados en la construcción de la comunidad. El modelo del NYT es similar al de la $B B C$ en la medida en que no permite los comentarios en todos sus artículos, pero, a diferencia de la cadena británica, no emplea a sus lectores como fuente y, por lo general, suele permitir comentarios en piezas más interpretativas o de opinión.

En el diario norteamericano, la participación ciudadana está basada en la opinión. Los usuarios hablan sobre diferentes temas y exponen sus argumentos. Las aportaciones son moderadas a priori y, a continuación, el medio destaca aquellos comentarios más enriquecedores, pero los periodistas no intervienen en las discusiones. Se trata, en definitiva, de abrir vías a la participación, pero sin una gestión para la incorporación de las aportaciones de los ciudadanos en la construcción del cuerpo de las piezas informativas.

El modelo del diario norteamericano descansa sobre una estructura muy vertical, en la que los periodistas tienen como cometido la elaboración de la información y el 
papel de los usuarios se limita a hablar sobre esas informaciones, aunque con poca interacción. Un aspecto en el que ha avanzado el NYT es en permitir que los usuarios que prueban su respeto por las normas de la comunidad publiquen directamente sus comentarios, ahorrando tiempo y mostrando cierta confianza en sectores de la comunidad que dan muestras de responsabilidad en sus actuaciones.

\subsection{Algunos rasgos de cibermedios franceses y norteamericanos}

El análisis de cibermedios norteamericanos y franceses, realizado para la verificación de similitudes y de diferencias con el modelo sólido de la $B B C$, nos ha permitido descubrir diversidad de caminos para dar voz a las audiencias y aprovechar su potencial en la construcción del relato informativo. La mayoría de las vías están en fase de experimentación y, en general, obedecen al planteamiento informativo de cada medio y a su concepción del papel que deben desempeñar sus usuarios, así como su idea de la implicación del medio en la comunidad y las opciones para hacer realidad esta implicación.

En el caso de Francia ${ }^{7}$, el análisis constata la existencia de dos formas de gestión de la participación ciudadana, la primera, que busca una colaboración entre periodistas profesionales y los ciudadanos, estos últimos en calidad de fuentes, y la segunda, aquella que pretende generar debate y opinión. En los cibermedios Franceses analizados, en AgoraVox y Rue89 colaboran periodistas profesionales y ciudadanos, aunque se le otorga al ciudadano un mayor grado de participación. Con respecto al segundo modelo, podemos incluir Le Fígaro y Atlantico. A caballo entre estos dos modelos se encuentra Mediapart, con su división en dos partes, Le Journal, que crea debate y opinión con sus artículos escritos por profesionales, y $L e C l u b$, que permite a los abonados crear sus propios artículos funcionando como fuentes de información pero también como "periodistas". Una fragmentada visión de la participación ciudadana, pues, a pesar de que las voces de los ciudadanos son escuchadas y reproducidas a través de los artículos que ellos mismos pueden escribir, estos se separan físicamente de las informaciones redactadas por los periodistas profesionales (al margen de los comentarios que se pueden publicar bajo cada artículo del diario).

AgoraVox desarrolla una estrategia de implicación de los usuarios en el producto permitiéndoles crear sus propios artículos, como también lo hace Mediapart y Rue 89, previa moderación. Otra opción es la que siguen Atlantico y Le Figaro, que limitan la participación a la aportación de comentarios en los foros que se crean bajo cada artículo. Tanto AgoraVox como Rue 89 permiten tanto redactar como comentar y gestionar la información. Por su parte, Mediapart, para buscar la colaboración entre profesionales y no profesionales, decidió dividir en dos su diario, con una primera mitad dedicada a los artículos redactados por periodistas que los usuarios pueden comentar y valorar, y otra mitad destinada a la participación de los abonados, que pueden crear sus propios contenidos.

7 El análisis de los cibermedios franceses, realizado por Carolina da Silva, incluye AgoraVox, Mediapart, Atlántico, Mediapart y Rue89. La investigación se enmarcó en un trabajo de fin de grado realizado en colaboración con el grupo Novos Medios. El trabajo se realizó en julio de 2013. 
En cuanto a las encuestas sobre los temas de actualidad, mientras que Atlantico, AgoraVox, Le Figaro y Rue89 ofrecen encuestas participativas sobre temas de actualidad, Mediapart va un paso por delante, ofreciendo además de éstas, una edición participativa en la que el único tema a tratar es el funcionamiento de la página. En ella, los abonados sugieren mejoras del sitio, aprueban o desaprueban nuevos proyectos o simplemente comentan acerca del funcionamiento de la página.

Sobre los espacios para que los usuarios sugieran temas, AgoraVox dispone de una sección, Tribune Libre, donde los usuarios pueden enviar artículos sobre temas que no tengan que ver con la estricta actualidad. En el apartado Le Club de Mediapart solo existen dos requisitos para publicar: estar abonado y que el equipo de Mediapart considere que la publicación del tema del artículo así como su contenido estén periodísticamente justificados, es decir, que sean pertinentes y originales. Con respecto a la parte de Le Journal, pueden ser sugeridos nuevos contenidos e incluso sugerencias en la edición participativa, Découvrez Mediapart. Con respecto a Rue89, la libertad de enviar contenidos es prácticamente ilimitada; solo hay que ponerse en contacto con el medio, previo registro y haciendo clic en Contacter á nous. Finalmente, Le Figaro y Atlantico, no ofrecen la posibilidad de sugerir contenidos.

En cuanto a los medios norteamericanos ${ }^{8}$, el Huffington Post dispone de un proyecto muy elaborado de participación ciudadana, dando un gran protagonismo a la comunidad en la generación de contenidos. Los ciudadanos participan en un intenso debate público que guían un modelo establecido por el propio medio, lo que le ha permitido construir una comunidad virtual donde los usuarios interactúan unos con otros, supervisados siempre por un equipo de periodistas profesionales. Los periodistas del Huffintton Post filtran los comentarios y destacan aquellos que consideran más relevantes.

Del análisis de ProPublica, destacaremos que tiene organizados los comentarios en dos secciones diferenciadas: la de los reportajes de investigación y los proyectos colaborativos de "Get Involved". En los reportajes, los usuarios pueden comentar y acercar opiniones, pero no hay interacción entre los periodistas y los ciudadanos. En este apartado, no hay una sección de destacados ni una estructura desarrollada para los comentarios. En los proyectos de "Get Involved", los ciudadanos actúan como fuentes, para lo que tienen que proporcionar ciertos datos y facilitar la información de la que disponen para su difusión.

El caso de The New Yorker es una muestra de una concepción del periodismo que apenas establece vías para la incorporación de los usuarios a su espacio web. El medio permite los comentarios en sus piezas y los modera para eliminar los que considera ofensivos. Pero no abre vías para el debate y la interacción, al tiempo que no establece mecanismos para su aprovechamiento como fuentes o para darles voz en las piezas informativas.

${ }^{8}$ El análisis de los medios norteamericanos lo realizó Pablo Mayo. Los medios estudiados de los que incluimos datos en este trabajo han sido Huffington Post, ProPublica, The New Yorker, como tres modelos diferenciados.El trabajo se realizó en julio de 2013. 


\section{A modo de conclusión}

Los resultados de las investigaciones y análisis de caso que se recogen en este texto constatan la existencia de relevantes aportaciones de ciudadanos interesados en contribuir a una buena información al servicio de la comunidad y cómo el buen trabajo de los periodistas puede evitar la difusión de datos imprecisos o informaciones incompletas sobre temas conflictivos de la actualidad si el modelo elegido para la participación es sólido y contempla acertadamente la intervención profesional.

La apertura de vías de participación es muy importante en los cibermedios actuales, pero sobre todo resulta trascendente y redunda en la calidad de los contenidos cuando el modelo favorece no solo los testimonios y los puntos de vista, sino, sobre todo, cuando garantiza la verificación por parte de los periodistas de todas las aportaciones recibidas y cuando en la redacción final de la pieza informativa se aplican criterios periodísticos.

Los responsables de los dos cibermedios analizados en el estudio de caso reconocen que la interacción con los ciudadanos ofrece altas cuotas de rentabilidad informativa y social, aunque consideran que resulta imprescindible no renunciar a la aplicación de la preceptiva periodística y, por tanto, al cumplimiento de los deberes del periodista a la hora de elaborar el mensaje y de gestionarlo en la sociedad en red. La existencia de un planteamiento periodístico en los dos casos, aunque con modelos formalmente diferentes, constituye una de las fortalezas de las dos experiencias. Los responsables de los dos medios entienden que deberán actualizar sus modelos y adaptarlos a las nuevas necesidades y a los nuevos desafíos.

Las conclusiones provisionales de esta primera aproximación a la cuestión de la participación actual en algunos cibermedios de referencia, tanto de los promovidos por medios tradicionales como algunos nativos digitales, apuntan a la necesidad de buscar renovadas vías para que los medios y los profesionales dispongan de las herramientas más innovadoras para un buen aprovechamiento de lo que algunos autores denominan inteligencia colectiva con una buena aplicación de lo que los defensores de la excelencia del periodismo identifican como los elementos básicos del periodismo de calidad. Como se ha constatado los medios analizados en este estudio de caso (BBC, The New York Times y The Guardian) conciben que la verificación de la información suministrada por sus usuarios se debe realizar a partir de una combinación de técnicas humanas y una serie de preceptos básicos que los periodistas deben tener en cuenta. Los tres cibermedios elegidos priorizan cuatro herramientas básicas de verificación de los contenidos: procedencia, localización de fuentes, fecha del material enviado y localización.

La participación de los usuarios en los cibermedios, tanto como emisores de opinión como de generadores de contenido informativo, es una realidad y se ha convertido en uno de los desafíos de los principales cibermedios. El camino de la experimentación, con sistemas en construcción, domina el panorama de los medios analizados. En estas primeras prácticas impera, como pudimos observar, la representación visual de datos a través de herramientas de geolocalización (tal y como muestra el caso del funeral de Margaret Thatcher de la $B B C$ y el registro de muertes de la guerra de Irak por The Guardian) o la utilización de vídeos como principal fórmula 
narrativa de los acontecimientos como se manifiesta las historias publicadas a partir de contenido generado por los usuarios del medio estadounidense The New York Times.

Con todo, los modelos actuales ya nos ofrecen lecciones aprovechables para, en el campo de la práctica ciberperiodística, combinar acertadamente participación de las audiencias con calidad periodística.

\section{Referencias bibliográficas}

AVILÉS, José Alberto. y CARVAJAL, Miguel (2008): “Integrated and Cross-Media Newsroom Convergence: two models of multimedia news production. The cases of Novo Técnica and La Verdad Multimedia in Spain”. Convergence, 14 (2), pp. 221-239.

BOCZKOWSKI, Pablo (2006): Digitalizar las noticias. Buenos Aires, Manantial.

BRETZ, Rudy (1983): Media for interactive communication. Londres, Sage.

CANAVILHAS, Joao (2011): "Do gatekeeping ao gatewatcher: o papel das redes sociais no ecossistema mediático". Puede consultarse en: http://campus.usal.es/ comunicacion3punto0/comunicaciones/061.pdf [Fecha de consulta: 12 de noviembre de 2013]

DAHLGREN, Peter (2012): "Mejorar la participación: la democracia y el cambiante entorno de la web". En CHAMPEAU, Serge e INNERATY, Daniel (comps.), Internet y el futuro de la democracia. Barcelona, Paidós.

DEUZE, Mark (2005): “Towards profesional participatory. Storytelling: mapping the potencial". Puede consultarse en: http://web.mit.edu/comm-forum/mit4/papers /Deuze.pdf [Fecha de consulta: 12 de noviembre de 2013].

DOMINGO, David (2007): “Audiencia activa: una minoría significativa”. Infotendencias. Puede consultarse en: http://infotendencias.com/2007/05/15/audencia-activa-una-minoria-significativa/ [Fecha de consulta: 12 de noviembre de 2013].

DOMINGO, David.; QUANDT, Thorsten.; HEINONEN, Ari.; PAULUSSEN, Steve.; SINGER, Jane.; \& VUJNOVIC, Marina (2007): "Participatory journalism practices in the media and beyond: an international comparative study of initiatives in online newspapers. Paper presentado en la Conferencia sobre el Futuro de los Periódicos. Cardiff, septiembre 2007.

GARCÍA DE TORRES, Elvira (2012): Cartografía del periodismo participativo. Valencia, Tirant Humanidades.

ITURBE, Mikel (2012): La participación ordenada del público en los medios de comunicación. El caso de BBC.co.uk. (Tesis doctoral). Pamplona, Universidad de Navarra.

JENSEN, Jens F. (1998): "Interactivity. Tracking a new concept in media and communication studies". Nordicom Review, 19 (1), pp.185-204.

MCKENZIE, Pamela J., BURKELL, Jacquelyn, WONG, Lola, WHIPPEY, Samuel E. y MCNALLY, Michael B. (2012): User-generated online content 1: Overview, cur- 
rent state and context. En: http://firstmonday.org/htbin/cgiwrap/bin/ojs/index.php /fm/article/viewArticle/3912/3266 [Fecha de consulta: 24 de febrero de 2014].

MASIP, Pere; DÍAZ NOCI, Javier; DOMINGO, David; MICÓ-SANZ, Josep-Lluís, y SALAVERRÍA, Ramón (2010): "Investigación internacional sobre ciberperiodismo: hipertexto, interactividad, multimedia y convergencia". El Profesional de la Información, 19, número 6.

MAZZONE, Daniel (2012): Huffington Post vs. New York Times. ¿Qué ciberperiodismo?. Buenos Aires, La Crujía Ediciones.

ORIHUELA, José Luis (2008): "Gestión de comentarios en cibermedios". En Ecuaderno. Puede consultarse en: http:/www.ecuaderno.com/2008/10/10/gestion-decomentarios-en-cibermedios [Fecha de consulta: 12 de noviembre de 2013].

ÖRNEBRING, Henrik (2008): "The Consumer as Producer - of What? User-generated Tabloid Content in The Sun (UK) and Aftonbladet (Sweden)". Journalism Studies, 9(5), pp. 771-785.

PAULUSSEN, Steve y UGILLE, Pieter (2008): "User-generated Content in the Newsroom: Professional and Organisational Constraints on Participatory Journalism". Communication and Culture 5(2), pp. 24-41.

PRESTON, Jennifer (2013): “A rapid biography of Jennifer Preston”. Entrevista en The Myndset: http://themyndset.com/2013/03/social-media-editor-jennifer-preston-at-nyt [Fecha de consulta: 24 de febrero de 2014].

RAIMONDO, Natalia (2012): La prensa online y su público. Un estudio de los espacios de intervención y participación del lector en Clarín y La Nación. Buenos Aires, Teseo.

ROST, Alejandro (2006): La interactividad en el periódico digital. (Tesis doctoral). Barcelona, Universidad Autónoma.

SALAVERRÍA, Ramón y GARCÍA AVILES, José Alberto (2008): "La convergencia tecnológica en los medios de comunicación: retos para el periodismo". Revista Trípodos, 22, pp. 31-47.

SALAVERRÍA, Ramón (2012). “Abre los comentarios y tápate la nariz”. En el blog Comunicando (25 de septiembre de 2012). Disponible en: http://blogs.unir.net/comunicacion/2012/09/25/abre-los-comentarios-y-tapate-la-nariz/. [Fecha de consulta: 28 de enero de 2013].

STARK, Birgit y LUNT, Peter (2012): "Public voice and mediated participation". The European Journal of Communication Research, 37, número 3.

SILVERMAN, Craig (2013): "Verification Handbook". Disponible en: http://verificationhandbook.com/book/ EE.UU: Silverman.

SUAU, Jaume (2011): "La participación en los medios digitales". En VI Congrés Internacional Comunicació i Realitat. Facultad de Comunicación BlanquernaRamón Llul. Trípodos Extra. 
TURNER, David (2012): "Inside the BBC's Verification Hub". En: http://www.nieman.harvard.edu/reports/article/102764/Inside-the-BBCs-Verification-Hub.aspx [Fecha de consulta: 24 de febrero de 2014].

THURMAN, Neil (2008): "Forums for citizen journalists?. Adoption of user generated content initiatives by online news media". New media\&Society, 10 (1), pp. 139-157.

VEHKOO, Johanna (2013): “Crowdsourcing in Investigative Journalism". Reuters Institute for the Study of Journalism.

WARDLE, Claire y WILLIAMS, Andy (2011): "Beyond user-generated content: a production study examining the ways in which UGC is used at the BBC". Media, Culture\&Society, 32 (5), pp. 781-799. 